La vulnerabilidad externa de la economía colombiana en el periodo 1990-2015: un análisis comparativo

Luis Hernando Portillo-Riascos y Edinson Ortiz-Benavides 
La vulnerabilidad externa de la economía colombiana en el periodo 1990-2015: un análisis comparativo

Resumen: El objetivo de este estudio es cuantificar la vulnerabilidad externa de la economía colombiana para el lapso 1990-2015. Para alcanzar este propósito, se calcularon dos indicadores, tomando como referencia la propuesta de Abeles y Valdecantos (2016). A partir de los resultados encontrados, se evidenció que, durante el transcurso del periodo estudiado, la economía colombiana, durante el transcurso del periodo estudiado, incrementó tanto su vulnerabilidad real como la financiera. Frente a este halla ago, se argumentó que la mayor vulnerabilidad externa de este país se explica por dos razones: a) la incapacidad de esta economia para modificar su modelo de inserción comercial basado en la explotación de los recursos naturales y b) el incremento de la dependencia del financiamiento externo. Palabras clave: vulnerabilidad real; vulnerabilidad financiera; vulnerabilidad externa.

Clasificación JEL: F40, O10, O54, O13.

The External Vulnerability of the Colombian Economy 1990-2015: A Comparative Analysis

Abstract: The objective of this study is to quantify the external vulnerability of the Colombian economy from 1990 to 2015. To achieve this purpose, two indicators were calculated, taking the proposal of Abeles and Valdecantos (2016) as reference. The results proved that the Colombian economy, during the period studied, increased its real and financial vulnerability. Facing this finding, the argument is made that the country's greatest external vulnerability is explained for two reasons: (a) the inability of this economy to modify her model of commercial insertion based on the exploitation of natural resources and (b) the increase in dependence of the external financing.

Keywords: real vulnerability; financial vulnerability; external vulnerability.

https://doi.org/10.17533/udea.le.n94a339023

\section{(cc) BY-NC-SA}

Este artículo y sus anexos se distribuyen por la revista Lecturas de Economía bajo los términos de la Licencia Creative Commons Atribución-NoComercial-CompartirIgual 4.0. https://creativecommons.org/licenses/by-nc-sa/4.0/ 
La vulnérabilité extérieure de l'économie colombienne au cours de la période 1990-2015 : une analyse comparative

Résumé: Cette étude a pour but de quantifier la vulnérabilité externe de l'économie colombienne pour la période 1990-2015. Pour atteindre cet objectif, deux indicateurs ont été calculés, en prenant comme référence la proposition d'Abeles et Valdecantos (2016). Les résultats obtenus ont montré que, au cours de la période étudiée, l'économie colombienne a augmenté sa vulnérabilité réelle et financière. Au vu de ce constat, il a été avancé que la plus grande vulnérabilité extérieure de ce pays s'explique par deux raisons: a) l'incapacité de cette économie à modifier son modèle d'insertion commerciale basé sur l'exploitation des ressources naturelles et b) l'augmentation de la dépendance à l'égard des financements extérieurs.

Mots clés: vulnérabilité réelle; vulnérabilité financière; vulnérabilité extérieure.

Cómo citar / How to cite this item:

Portillo-Riascos, L. H. \& Ortiz-Benavides, O. (2021). La vulnerabilidad externa de la economía colombiana en el periodo 1990-2015: un análisis comparativo. Lecturas de Economia, 94, 11-46.

https://doi.org/10.17533/udea.le.n94a339023 


\title{
La vulnerabilidad externa de la economía colombiana en el periodo 1990-2015: un análisis comparativo
}

\author{
Luis Hernando Portillo-Riascos $\oplus^{a}$ y Edinson Ortiz-Benavides $\oplus^{b}$ \\ -Introducción. -I. Revisión de la literatura. -II. Metodología. -III. Resultados. \\ -Conclusiones. -Agradecimientos. -Referencias.
}

Primera versión recibida el 02 de julio de 2019; versión final aceptada el 10 de junio 2020

\section{Introducción}

La vulnerabilidad macroeconómica de un país se define como el grado de exposición de su economía ante la probabilidad de ocurrencia de un choque externo (external shock), y la capacidad de dicha economía para afrontar los efectos adversos que este genere (véase Atkins et al., 1998; Cordina, 2004; Briguglio et al., 2009; Guillaumont, 2013, 2016). La vulnerabilidad macroeconómica se origina tanto por factores externos como internos. Así, mientras el carácter externo se explica por la independencia y las características de los choques (Ffrench-Davis, 2008), el interno tiene que ver con el manejo que hacen los gobiernos de los instrumentos de política económica y las características de la estructura productiva de cada país (Prebisch, 1949; Ffrench-Davis, 2008, 2009; Ocampo, 2011, Portillo-Riascos, 2020a, 2020b).

Los choques externos, reflejados en las variaciones de los términos de intercambio, las oscilaciones de los flujos internacionales de capital y las modificaciones de las tasas de interés internacionales (Ffrench-Davis, 2008, 2009), han sido determinantes a la hora de explicar la dinámica macroeconómica de corto plazo de los países en desarrollo en las últimas

a Luis Hernando Portillo-Riascos: docente de tiempo completo adscrito al Departamento de Economía de la Universidad de Nariño, Colombia. Dirección electrónica: luiher02@ucm.es https:// orcid.org/0000-0003-3362-6934

b Edinson Ortiz-Benavides: docente de tiempo completo adscrito al Departamento de Economía de la Universidad de Nariño, Colombia. Dirección electrónica: edinson@udenar.edu.co https://orcid.org/0000-0002-2500-4177 
Portillo-Riascos y Ortiz-Benavides: La vulnerabilidad externa de la economía colombiana...

décadas, particularmente en el caso de las economías latinoamericanas (Thirlwall, 1979, 2011; Ocampo, 2011, 2012; Bielschowsky et al., 2011). Así pues, debido al alto grado de sensibilidad que muestran las economías de estos países ante los efectos que pueden generar los choques exógenos, el tema de la vulnerabilidad externa ha sido uno de los aspectos que más se ha destacado tanto en el estructuralismo latinoamericano como en el neoestructuralismo (Prebisch, 1949; Rodríguez, 2006; Ocampo, 2011, 2012; Comisión Económica para América Latina y el Caribe -CEPAL_, 2012).

Ahora bien, el término vulnerabilidad se ha utilizado recurrentemente en diferentes investigaciones, sin que haya la suficiente claridad acerca del significado del mismo (Chambers, 1989; Busso, 2001; Villagrán, 2006; Seth \& Ragab, 2012). Desde un punto de vista económico, dichas divergencias son evidentes, no solamente en el plano conceptual, sino también en el análisis empírico (Briguglio, 1995, 2014; Atkins et al., 1998; Guillaumont, 2010, 2016; Montalbano, 2011; Bates et al., 2014; Angeon \& Bates, 2015; Abeles \& Valdedantos, 2016; Valdecantos, 2016). En términos conceptuales, Seth y Ragab (2012) mencionan dos tipos de enfoques que se han utilizado a la hora de estudiar la vulnerabilidad macroeconómica. En primer lugar, citan aquellas investigaciones para las cuales la vulnerabilidad macroeconómica de un país representa un fenómeno estructural, asociado no solamente a problemas de tipo económico, sino también a aspectos geográficos, demográficos, ambientales, etc. (Briguglio, 1995, 2014; Atkins et al., 1998; Guillaumont, 2010, 2016; Portillo-Riascos, 2020a, 2020b). En segundo lugar, Seth y Ragab (2012) relacionan los enfoques a partir de los cuales la vulnerabilidad macroeconómica se entiende como un fenómeno de corto plazo, donde toman relevancia aquellos indicadores relacionados con la ocurrencia de algún tipo de crisis financiera (bancaria, cambiaria o de deuda), fiscal o real (Kaminsky et al., 1998; Fondo Monetario Internacional -FMI-, 1998, 2010, 2011; Herrera \& García, 1999; Ghosh \& Ghosh, 2003; Frankel \& Saravelos, 2012; Dabla-Norris \& Gündüz, 2014).

Frente al segundo grupo de enfoques, Seth y Ragab (2012) incluyen aquellas posturas a partir de las cuales la vulnerabilidad macroeconómica de un país se origina por las características que han mostrado los flujos internacionales de capital en las últimas décadas, y los efectos que 
generan, particularmente, en las economías en desarrollo, incluidas las latinoamericanas (Ffrench-Davis, 2010; Ocampo, 2011, Valdecantos, 2016, Abeles \& Valdecantos, 2016, Portillo-Riascos, 2020a, 2020b). En este último caso, los aportes provienen del estructuralismo latinoamericano y del neoestructuralismo. Ahora bien, a pesar de la trascendencia que tiene la vulnerabilidad externa en el campo teórico, las propuestas para la medición de esta variable desde estos enfoques han sido muy escasas.

Teniendo en cuenta los elementos señalados hasta el momento, en este estudio se cuantifica la vulnerabilidad externa de la economía colombiana para el lapso 1990-2015, tomando como referencia los planteamientos neoestructuralistas. Además, se hace un comparativo de los resultados obtenidos para este país con relación a otras economías. Para alcanzar este propósito, se calculan dos indicadores sintéticos propuestos por Abeles y Valdecantos (2016), quienes abordan dos aspectos que son fundamentales en el planteamiento neoestructuralista: la vulnerabilidad real y la vulnerabilidad financiera.

Este artículo está organizado en cuatro secciones, además de esta introducción. En la sección I se presenta un breve repaso de la literatura acerca de la vulnerabilidad externa; en la II se realizan las aclaraciones metodológicas; en la sección III se aborda el análisis empírico y, finalmente, se presentan las conclusiones más importantes que se obtuvieron con esta investigación.

\section{Revisión de la literatura}

La necesidad de cuantificar la vulnerabilidad externa se ha acrecentado durante las últimas décadas, especialmente por el incremento de la interdependencia comercial y financiera entre países. En este marco, se han propuesto diferentes metodologías para la cuantificación de la vulnerabilidad externa, a partir de las cuales se han realizado comparaciones tanto en términos temporales como entre grupos de países (véase Briguglio, 1995, 2014; Atkins et al., 1998; Guillaumont, 2010, 2016; Montalbano, 2011; Bates et al., 2014; Angeon \& Bates, 2015; Abeles \& Valdedantos, 2016; Valdecantos, 2016). Con todo, al haber grandes diferencias en la conceptualización de la vulnerabilidad macroeconómica, los métodos de medición también son 
Portillo-Riascos y Ortiz-Benavides: La vulnerabilidad externa de la economía colombiana...

disímiles. De cualquier forma, avanzar en este campo se muestra como una tarea de gran relevancia.

Guillaumont (2016) justifica la necesidad de medir la vulnerabilidad macroeconómica de un país por dos razones: porque es una herramienta que se puede utilizar para la asignación de recursos en el contexto internacional, y porque hay necesidad de identificar indicadores de riesgo de crisis, aunque advierte que esta es una tarea muy compleja.

Desde aquellos enfoques que asocian la vulnerabilidad macroeconómica con episodios de crisis financieras (cambiarias, bancarias o de deuda), la medición de la vulnerabilidad macroeconómica es necesaria debido a que permite identificar las variables que pueden anticipar un escenario de crisis, que pueden ser financieras, fiscales o reales (FMI, 1998, 2010, 2011; Kaminsky et al., 1998; Ghosh \& Ghosh, 2003; Frankel \& Saravelos, 2012). En este marco, las variables más utilizadas para la construcción de los modelos son las siguientes: PIB, producción industrial, tipo de cambio, índices del mercado bursátil, reservas internacionales y participación del país en un programa del FMI (Frankel \& Saravelos, 2012).

Ahora bien, a pesar de la solidez que han mostrado estos modelos en la explicación de determinados episodios de crisis, la principal crítica que se les ha planteado está relacionada con su baja capacidad predictiva (Frankel \& Saravelos, 2012). Esta situación se explica por dos razones (Ghosh \& Ghosh, 2003): en primer lugar, por el hecho de que el análisis se basa en episodios de crisis que ya sucedieron, y que no necesariamente se van a dar en las mismas condiciones hacia el futuro; $y$, en segundo lugar, por el uso de variables coyunturales, que, si bien es cierto que son muy importantes en términos estadísticos, no juegan un mismo rol en diferentes contextos y momentos.

A diferencia de los enfoques relacionados anteriormente, el neoestructuralismo ha resaltado la importancia de los factores estructurales. En este punto, se enfatiza en el rol que siguen desempeñando las asimetrías (productivas, financieras, macroeconómicas y de movilidad de la mano de obra) existentes entre las economías en desarrollo y los países desarrollados (Ocampo, 2001). Siguiendo esta línea, se concluye que la dinámica macroeconómica de este tipo de países se explica, básicamente, por la 
ocurrencia de choques externos, comerciales o financieros, tanto positivos como negativos (Ocampo, 2011, 2012).

Bajo el enfoque neoestructuralista, además de las variables coyunturales, y particularmente las financieras, toman importancia los factores reales, que están relacionados con el modelo de inserción comercial y financiera que han mantenido los países latinoamericanos a lo largo de su historia (véanse Ocampo, 2008, 2012; Ffrench-Davis, 2010, 2015; Bielschowsky et al., 2011). Así entonces, la principal consecuencia del alto grado de sensibilidad que muestran este tipo de países ante la ocurrencia de choques externos es la persistencia de la inestabilidad macroeconómica real, que se refleja en un crecimiento económico bajo y volátil. En este sentido, de lo que se trata no es de explicar un episodio de crisis como tal, sino de entender aquellos factores que ralentizan el crecimiento económico (Portillo-Riascos, 2020a).

Las propuestas para la medición de la vulnerabilidad externa desde los enfoques estructuralista y neoestructuralista son muy escasas. En términos generales, lo que se plantea es que la vulnerabilidad externa de un país se explica tanto por factores estructurales como coyunturales, los cuales afectan el grado de exposición al que están sometidas sus economías ante la ocurrencia de choques externos (Abeles \& Valdecantos, 2016).

Siguiendo los planteamientos neoestructuralistas, hay varios estudios que han buscado cuantificar los efectos de los choques externos sobre la dinámica macroeconómica de un país o grupo de países, lo que representaría una medida de vulnerabilidad externa. Para el caso latinoamericano, Loayza y Raddatz (2007) realizaron una investigación en la cual estiman la sensibilidad del PIB ante los choques reales, representados en las fluctuaciones de los términos de intercambio; los autores encontraron que los factores estructurales son determinantes para explicar la vulnerabilidad externa de un país. A diferencia de Loayza y Radattz (2007), Le Fort y Budnevich (2005) se enfocaron en evaluar el impacto de los movimientos cíclicos de la cuenta financiera en el crecimiento económico de varios países latinoamericanos.

Titelman et al. (2008) analizaron los efectos generados por los choques comerciales y los financieros sobre el ciclo económico de los países latinoamericanos durante el periodo 1980-2006, y encontraron que dichos 
Portillo-Riascos y Ortiz-Benavides: La vulnerabilidad externa de la economía colombiana...

choques han venido tomando mayor relevancia en las últimas décadas, particularmente por el comportamiento adoptado por los flujos financieros internacionales.

Por otra parte, Gonçalves et al. (2009, p. 10) proponen un índice de vulnerabilidad estructural externa - IVE - entendiendo que esta se "deriva de los cambios relativos al patrón de comercio, de la eficiencia del aparato productivo, del dinamismo tecnológico y de la robustez del sistema financiero nacional". A partir de los cuatro indicadores propuestos, estos investigadores calcularon el IVE para un conjunto de 113 países, incluidos varios latinoamericanos. El lapso estudiado va desde 1980 hasta 2006.

Siguiendo la línea de trabajo de Le Fort y Budnevich (2005), Da Costa y Libânio (2013) estimaron dos indicadores que permiten evaluar la vulnerabilidad externa de Brasil: la volatilidad de los flujos de capitales y una medida de fuga de capitales. Siguiendo dicha postura, la vulnerabilidad externa de un país está relacionada con la probabilidad de reversión de las entradas de capital, que a su vez está asociada con la composición de los mismos.

Por último, Abeles y Valdecantos (2016) desarrollaron un ejercicio empírico a partir del cual cuantificaron la vulnerabilidad externa de un conjunto de 32 países de América Latina y el Caribe para el periodo 20052015. El objetivo de esta investigación fue hacer un análisis comparativo del indicador de vulnerabilidad externa antes y después de la crisis financiera que se dio en varios países desarrollados durante el lapso 2008-2009. La ventaja que tiene esta propuesta es que permite hacer comparaciones de los resultados, tanto entre países como en términos temporales. En virtud de ello, en adelante, se hace una descripción más amplia de la misma.

Abeles y Valdecantos (2016) y la CEPAL (2016) sugieren dos indicadores que reflejan el grado de exposición de la economía de un país ante la ocurrencia de los choques externos. Bajo esta propuesta, las causas estructurales de la vulnerabilidad externa están determinadas por la especialización productiva de cada país, el grado y composición del endeudamiento externo y la acumulación de reservas internacionales. Dichos factores "resultan de una serie de circunstancias histórico-estructurales que 
determinan el tipo de riesgos a los que las economías de la región están expuestas" (Abeles \& Valdecantos, 2016, p. 19).

Así mismo, tanto los autores como la CEPAL definen dos indicadores de vulnerabilidad externa: uno real y otro financiero. Las vulnerabilidades reales de un país están determinadas por las fluctuaciones de los términos de intercambio o la variación del ritmo de crecimiento de los principales socios comerciales de cada país. Por su parte, las vulnerabilidades financieras están asociadas a las fluctuaciones de los flujos de inversión externa (de corto y largo plazo). Por tanto, la vulnerabilidad externa depende del grado de concentración de la estructura exportadora y la cantidad de socios comerciales, así como del apalancamiento externo. Este último factor determinaría la exposición de un país frente a las interrupciones repentinas de los flujos financieros internacionales o los cambios en las condiciones de financiación.

A partir de la propuesta de Abeles y Valdecantos (2016, p. 19) y la CEPAL (2016), el indicador de vulnerabilidad real (VR) se calcula como se muestra en la ecuación 1.

$$
V R_{t}^{i}=\operatorname{máx}\left(\frac{X_{t}^{P^{i}}}{\operatorname{Créditos}_{t}^{C A^{i}}} ; \frac{X_{t}^{M^{i}}+X_{t}^{V^{i}}+R_{t}^{i}}{\operatorname{Créditos}_{t}^{C A^{i}}}\right),
$$

donde $X^{P}$ representa las exportaciones intensivas en recursos naturales; $X^{M}$, las exportaciones de manufacturas; $X^{V}$, las exportaciones de servicios turísticos (viajes); $R$, la recepción de remesas, y Créditos ${ }^{C A}$ representa los créditos de la cuenta corriente (suma de ingresos de divisas por todo concepto, incluidas las exportaciones de bienes, servicios y remesas).

Para obtener el indicador de $V R$, se toma el porcentaje más alto que resulta del cociente entre las exportaciones de bienes primarios y la suma de los productos manufacturados, los servicios turísticos y las remesas; ambos divididos entre los créditos totales de la cuenta corriente. De esta forma, cuanto más alto sea dicho resultado, mayor es el nivel de vulnerabilidad real. $\mathrm{Al}$ respecto, es importante advertir que la vulnerabilidad externa de un país se genera tanto por la mayor concentración de las exportaciones en bienes de origen primario como por el incremento de la dependencia de las remesas, así como de actividades turísticas y, en el caso de las manufacturas, de los bienes 
Portillo-Riascos y Ortiz-Benavides: La vulnerabilidad externa de la economía colombiana...

relacionados con procesos como los de ensamblaje o maquila, en los cuales el valor agregado tiende a ser mínimo (Abeles \& Valdecantos, 2016).

Frente al indicador de vulnerabilidad financiera $(V F)$, se proponen dos opciones. En la primera, la VF se calcula siguiendo la ecuación 2.

$$
V F_{t}^{i}=\frac{I C_{t}^{i}+I E D_{t}^{i}-\operatorname{Reservas}_{t}^{i}}{P I B_{t}^{i}},
$$

donde $I C$ son los pasivos del stock de inversiones de cartera; $I E D$, los pasivos del stock de inversión extranjera directa; Reservas presenta los stock de reservas internacionales del banco central y $P I B_{t}^{i}$ el Producto Interno Bruto.

Dado que la información acerca del stock de inversión de cartera $(I C)$ no está disponible para todos los países, esta variable se puede reemplazar por el stock de deuda externa pública (Abeles \& Valdecantos, 2016). De esta forma, el indicador de VF también se puede expresar de la siguiente manera:

$$
V F_{t}^{i}=\frac{D E P_{t}^{i}+I E D_{t}^{i}-\operatorname{Reservas}_{t}^{i}}{P I B_{t}^{i}},
$$

donde $D E P$ es el stock de la deuda externa pública bruta.

De acuerdo con el indicador de $V F$, en cualquiera de las dos propuestas, la vulnerabilidad externa de un país será mayor cuanto más alto es el nivel de apalancamiento externo, medido por la ratio entre el stock de la deuda externa pública (o la $I C$ ) y el stock de Inversión Extranjera Directa-IED-con respecto al $P I B$. En contraste, la $V F$ se reduce cuanto mayor es el cociente entre el stock de reservas internacionales y el PIB. En este caso, se supone que, al incrementarse las reservas internacionales, el grado de exposición de la economía de un país ante episodios de salida masiva de capitales es menor.

\section{Metodología}

El objetivo de este estudio es cuantificar la vulnerabilidad externa de la economía colombiana para el periodo 1990-2015 y realizar una comparación de los resultados obtenidos para Colombia con respecto a otros países. Los 
cálculos se hacen siguiendo la propuesta de Abeles y Valdecantos (2016). Esta decisión se toma por dos razones: porque las variables que se utilizan corresponden a aspectos estructurales de la vulnerabilidad externa, que han sido resaltados tanto en el estructuralismo latinoamericano como en el neoestructuralismo, y porque la información disponible permite hacer contrastes entre países.

Como se mencionó en la introducción, para el análisis de la vulnerabilidad externa desarrollado dentro de esta investigación se utilizan indicadores sintéticos o compuestos. Conceptualmente, "un indicador compuesto es una representación simplificada que busca resumir un concepto multidimensional en un índice simple (unidimensional) con base en un modelo conceptual subyacente" (Schuschny \& Soto, 2009, p. 13). De acuerdo con la clasificación señalada por estos autores, la medida utilizada en la presente investigación corresponde a un indicador compuesto de tipo sinóptico.

Metodológicamente, Schuschny y Soto (2009) argumentan la utilidad de este tipo herramientas en diversos campos del conocimiento, incluyendo el económico, debido a su capacidad de síntesis, la facilidad de interpretación de los resultados, la posibilidad de hacer comparaciones (benchmark) entre distintas unidades de análisis (e. g. países) y el seguimiento de los valores reportados en distintos momentos del tiempo (evolución). Al final, la principal ventaja de un indicador sintético es la de resumir la información proveniente de varias dimensiones y que a su vez están relacionadas.

Para Schuschny y Soto (2009), un indicador sintético debe cumplir tres criterios: sustento conceptual, validez y sustento técnico. En esta investigación dichos requisitos se cumplen. En primer lugar, porque está definido el enfoque teórico que soporta cada una de las variables seleccionadas. En segundo lugar, porque la información se toma de fuentes de datos de libre acceso, lo cual facilita la replicabilidad de los cálculos. Y, en tercer lugar, porque se expone el método específico que permite calcular cada uno de los indicadores que hacen parte de la vulnerabilidad externa.

Sobre el uso de indicadores sintéticos en el análisis de la vulnerabilidad macroeconómica, Guillaumont (2009) y Briguglio et al. (2009) destacan varias ventajas. Para los autores, aunque se podría estar dejando por fuera 
Portillo-Riascos y Ortiz-Benavides: La vulnerabilidad externa de la economía colombiana...

muchas variables, lo importante es capturar los aspectos estructurales de la vulnerabilidad y expresarlos fácilmente en un indicador. Dentro de los principios para escoger las variables y el diseño del instrumento de medición estarían los siguientes: simplicidad, transparencia, posibilidad de hacer comparaciones internacionales, fácil comprensión, acceso a la información, etc. (Briguglio, 1995, 2003; Briguglio et al., 2009; Guillaumont, 2009). Para Guillaumont (2016), los indicadores de vulnerabilidad macroeconómica deben seguir un criterio comparativo, a nivel de países, que es donde están la mayor parte de los trabajos en el campo empírico, además de permitir examinar los cambios en el tiempo.

Desde luego, el uso de indicadores sintéticos presenta algunas desventajas que es necesario señalar (Schuschny \& Soto, 2009). Para los propósitos de esta investigación, son importantes por lo menos dos aspectos. En primer lugar, una debilidad importante de los indicadores compuestos tiene que ver con la exagerada reducción de la información a unas pocas variables e indicadores, que además pueden recopilar información de diferentes dimensiones (Schuschny \& Soto, 2009). El segundo aspecto está relacionado con las ponderaciones que se realizan al momento de hacer los cálculos parciales o definitivos de los subindicadores o indicadores sintéticos. Lo que se ha argüido en este punto es que dicho procedimiento tiende a ser subjetivo, lo cual afectaría el peso real que puede tener cada variable en el fenómeno global que se pretende explicar (Atkins et al., 1998; Essers, 2013; Bates et al., 2014; Angeon \& Bates, 2015). De ahí que se sugiere, en algunos casos, que dichas ponderaciones sean establecidas a través de algún procedimiento estadístico (Schuschny \& Soto, 2009; Domínguez et al., 2011: Bates et al., 2014; Angeon \& Bates, 2015).

Haciendo un balance entre las ventajas y las desventajas del uso de los indicadores compuestos para los propósitos de esta investigación, se resalta que, a pesar de perder profundidad en la comprensión de la situación de la variable examinada en cada uno de los países — algo que se podría abordar con la incorporación de más variables y el uso de otros instrumentos estadísticos, lo más importante es que dicha herramienta ofrece la posibilidad de hacer comparaciones entre países y hacer un seguimiento a los cambios temporales. 
Volviendo a los aspectos metodológicos, para realizar el cálculo de los indicadores de vulnerabilidad externa utilizados dentro de esta investigación, se tomó la información de 14 países: Colombia, Chile, Argentina, Brasil, Uruguay, Costa Rica, Jamaica, India, Turquía, Arabia Saudita, Indonesia, Nigeria, Botswana y Argelia. La selección de los mismos fue arbitraria y se hizo teniendo en cuenta varios factores. En primer lugar, se consideró la estructura de inserción comercial, por lo que se tomaron aquellos países cuyas características son similares a las de la economía colombiana; en segundo lugar, la proximidad geográfica; y, por último, se tomaron algunos países donde del modelo de especialización productiva es totalmente diferente al caso colombiano.

Sobre los datos requeridos para el desarrollo de este estudio, es importante advertir que, a pesar de que la información para el cálculo de los indicadores de vulnerabilidad externa está disponible para la mayoría de países, en ciertos casos se presentaron algunas dificultades. Los principales inconvenientes estuvieron en el stock de inversión de cartera (IC), ya que la mayor parte de los datos están disponibles desde el 2000 en adelante. En estos casos, siguiendo la propuesta de Abeles y Valdecantos (2016), se utilizó el stock de la deuda externa pública. También se encontraron algunas limitaciones con los datos de las exportaciones de bienes primarios y los manufacturados. En el caso de los países de América Latina y el Caribe, la información la proporciona la CEPAL, a partir de la base de datos de la COMTRADE. Para el resto de países, la información no está disponible ${ }^{1}$. Ante esta situación, se optó por sumar las exportaciones de alimentos, minerales y combustibles (respecto del total de exportaciones de mercaderías). En el caso de las manufacturas, el cálculo se hizo utilizando la proporción de estas con respecto al total de exportaciones de mercaderías ${ }^{2}$.

1 Los datos se pueden encontrar en la web de la UNCTAD, pero solamente a partir de 1995. Se decidió trabajar con las estadísticas del Banco Mundial para poder homogeneizar la información de todo el periodo.

2 La información se encuentra disponible en la base de datos del Banco Mundial (World Development Indicators -WDI-) 
Portillo-Riascos y Ortiz-Benavides: La vulnerabilidad externa de la economía colombiana...

\section{Resultados}

Una vez se realizó el cálculo de los dos indicadores de vulnerabilidad externa, lo que se encontró fue que tanto la VR como la VF se incrementaron en la mayoría de los países seleccionados (Tabla 1). En el contexto sudamericano, Colombia, después de Chile, fue el país que reportó mayores variaciones (positivas) en los dos indicadores.

Tabla 1. Variación de la vulnerabilidad externa (2000-2015 respecto a 1990-1999)

\begin{tabular}{lclc}
\hline \multicolumn{2}{c}{ Vulnerabilidad real } & \multicolumn{2}{c}{ Vulnerabilidad financiera } \\
\hline Botswana & $830,28 \%$ & Sudáfrica & $176,63 \%$ \\
Nigeria & $43,30 \%$ & Chile & $167,33 \%$ \\
Sudáfrica & $35,09 \%$ & Uruguay & $147,68 \%$ \\
Turquía & $20,14 \%$ & Turquía & $103,38 \%$ \\
Costa rica & $15,23 \%$ & Colombia & $84,82 \%$ \\
Chile & $9,67 \%$ & Brasil & $82,86 \%$ \\
Colombia & $8,41 \%$ & Argentina & $56,72 \%$ \\
Jamaica & $6,43 \%$ & Jamaica & $24,29 \%$ \\
Argelia & $3,10 \%$ & Costa rica & $16,19 \%$ \\
Argentina & $2,67 \%$ & Indonesia & $-38,50 \%$ \\
Indonesia & $-2,48 \%$ & Nigeria & $-76,51 \%$ \\
Brasil & $-7,41 \%$ & India & $-94,75 \%$ \\
Uruguay & $-11,31 \%$ & Botswana & $-101,10 \%$ \\
India & $-18,58 \%$ & Argelia & $-200,06 \%$ \\
\hline
\end{tabular}

Fuente: elaboración propia con información del Banco Mundial, el FMI y las estadísticas oficiales de cada país.

Respecto a la vulnerabilidad externa los datos reportados en la Tabla 1 evidencian dos características para el caso colombiano. En primer lugar, muestran que dicho fenómeno ha sido relevante para esta economía en comparación con el resto de los países seleccionados, y, en segundo lugar, dejan ver que hay un incremento tanto de la VR como de la VF. Desde luego, para un mayor entendimiento de estos indicadores, es necesario profundizar en los componentes que hacen parte de ellos. Dicho ejercicio se desarrolla a continuación. 


\section{A. Vulnerabilidad real (VR)}

A partir de los datos encontrados para este indicador, se concluye que el crecimiento de la VR en los países sudamericanos se dio, fundamentalmente, por la mayor participación de los bienes primarios dentro de la estructura de las exportaciones; la cual se fortaleció desde mediados de la década de los 2000. Fruto del comportamiento de este tipo de ingresos, se pudo observar una reducción de la participación de las manufacturas, servicios turísticos y remesas. Este fenómeno también se dio en un país como la India, donde las manufacturas tienen un peso significativo dentro de la estructura de exportaciones. Algo similar sucedió en Brasil, Costa Rica, India y Turquía.

En la Figura 1 se presenta un comparativo de la participación de los ingresos por exportaciones de bienes primarios en los créditos totales de la cuenta corriente. Cuando el indicador se ubica por encima de la diagonal significa que hay un mayor peso respecto al periodo previo.

En el caso de Colombia, se incrementó la participación de los ingresos derivados de las exportaciones de bienes primarios dentro de los créditos totales de la cuenta corriente, y se pasó de un porcentaje promedio del 48,2\% entre 1990 y 1999 al 51,3\% entre 2000 y 2015 (Figura 1). Incluso, durante el periodo del boom de las commodities (2005-2013), dicho indicador se ubicó en el 53,7\%. A partir de estos resultados, queda claro que en la economía colombiana ha prevalecido un modelo de inserción comercial basado en bienes primarios. Dentro de los países seleccionados, a Colombia solamente la superan algunos países con una estructura muy concentrada en este tipo de bienes (Arabia Saudita, Argelia y Nigeria) y dos países sudamericanos con modelo de especialización productiva bastante similar, especialmente en el caso de Chile.

Con relación al segundo indicador de VR, en el que se incorporan los ingresos de las exportaciones de manufacturas, los servicios de turismo y las remesas, lo que se hizo fue separar la información de cada una de las variables con el fin de tener una mejor lectura de ellos. En el primer caso, hay una crítica en la construcción del indicador propuesto por Abeles y Valdecantos (2016), puesto que este no diferencia el tipo de manufacturas. Así, aunque se entiende que desde la CEPAL lo que se intentó fue mostrar 
Portillo-Riascos y Ortiz-Benavides: La vulnerabilidad externa de la economía colombiana...

la dependencia de algunos países —especialmente los centroamericanosrespecto a determinados tipos de bienes industriales que se exportan, pero que tienen un bajo contenido agregado, es evidente que esto no va a suceder en todas las situaciones, como pasa con Colombia. Dentro de este estudio, el mayor peso de las manufacturas se entiende como un aspecto positivo, en la medida que permite avanzar hacia la diversificación de las exportaciones, lo que a su vez reduciría la participación de los bienes primarios dentro de la estructura de inserción comercial.

Figura 1. Participación de los ingresos por exportaciones de bienes primarios en los créditos totales de la cuenta corriente

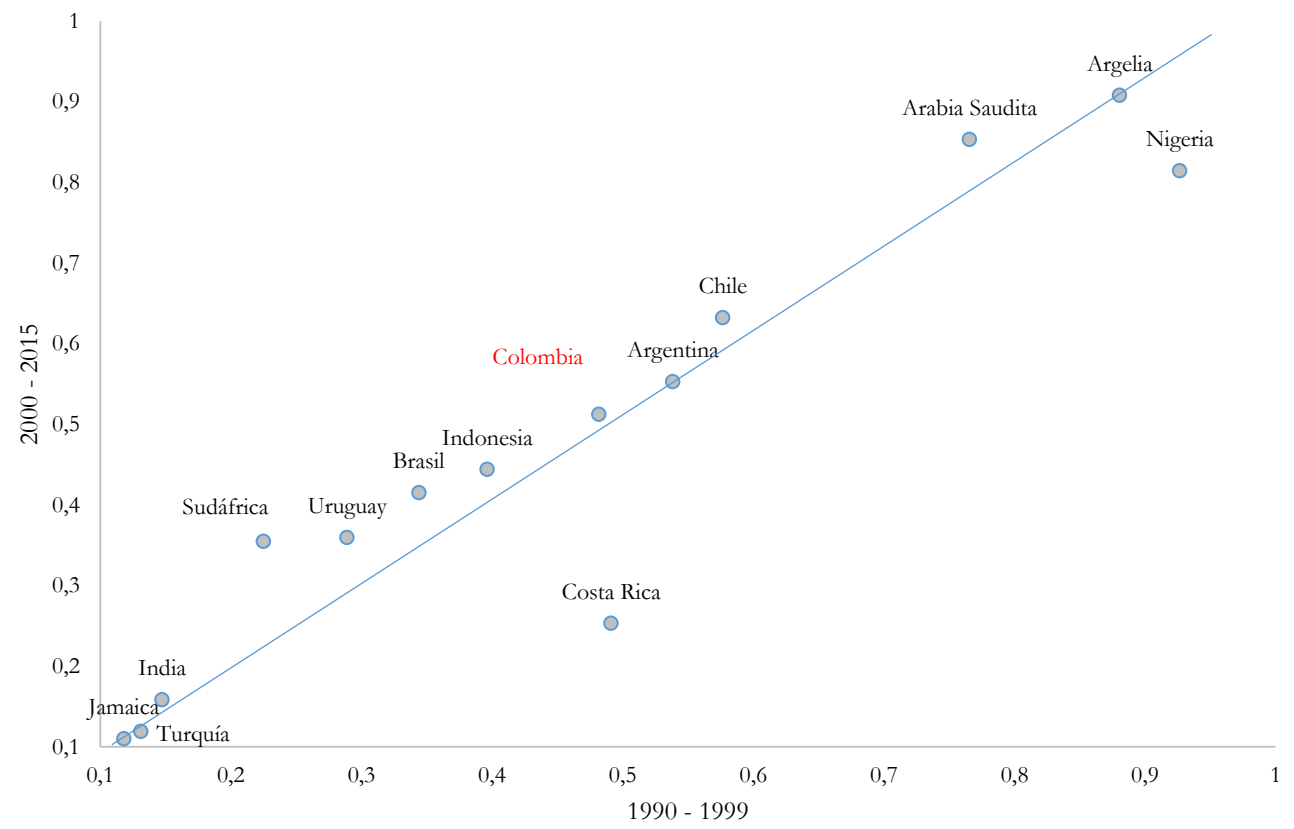

Fuente: elaboración propia con información de la CEPAL, el Banco Mundial, el FMI y las estadísticas oficiales de cada país.

Realizada esta aclaración, se determinó la participación de los ingresos derivados de las exportaciones de manufacturas en los créditos totales de la cuenta corriente para el conjunto de países analizados. Como se puede observar en la Figura 2, la mitad de los países seleccionados redujeron dicha 
ratio, y los que la aumentaron lo hicieron en un porcentaje mínimo. En el caso colombiano sucedió algo similar, aunque no hay mayores cambios entre los dos subperiodos analizados. Así, Colombia pasó de una ratio del 22,6\% en el subperiodo 1990-1999 a un porcentaje del 21,6\% entre 2000 y 2015 , el cual se redujo hasta el 14,9\% entre 2010 y 2015. A partir de estos resultados, nuevamente se concluye que Colombia no ha mostrado cambios relevantes en su modelo histórico de inserción comercial basado en la explotación de recursos naturales.

Figura 2. Participación de los ingresos por manufacturas en los créditos totales de la cuenta corriente

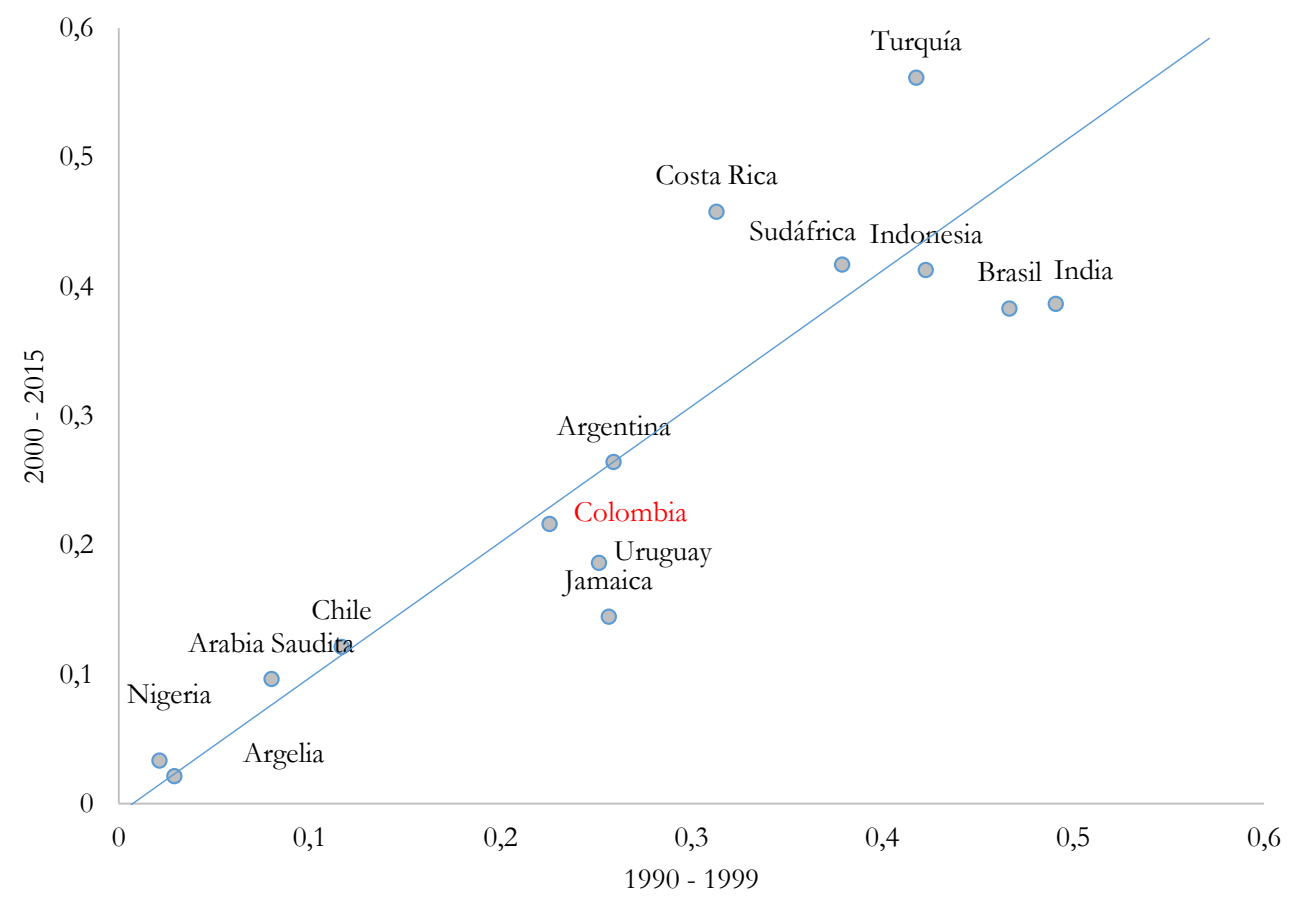

Fuente: elaboración propia con información de la CEPAL, el Banco Mundial, el FMI y las estadísticas oficiales de cada país.

El análisis de las exportaciones de manufacturas también se puede hacer a partir de su clasificación por categorías tecnológicas y aquellas que están basadas en recursos naturales (véase Lall, 2000). Dicha información es 
Portillo-Riascos y Ortiz-Benavides: La vulnerabilidad externa de la economía colombiana...

importante porque da cuenta del tipo de manufacturas que está exportando un país, lo cual permite profundizar en la evaluación del modelo de inserción comercial de cada economía. Desde el enfoque abordado dentro de este estudio, el tránsito hacia las exportaciones de manufacturas con un mayor contenido tecnológico indica el camino correcto hacia la reducción de la vulnerabilidad externa de un país.

Por categorías tecnológicas, el cambio más relevante que se dio en el subperiodo 1995-2015 para el conjunto de países analizados es la mayor participación de las manufacturas de tecnología media, en detrimento de las exportaciones de tecnología baja (Tabla 2). De hecho, mientras en 1995 las exportaciones de tecnología baja representaban el 51,6\% de este grupo, en 2015 dicho porcentaje fue del $28,9 \%$. En contraste, los porcentajes para las manufacturas de tecnología media fueron del $42 \%$ y $57,7 \%$, respectivamente. En la categoría de manufacturas de tecnología media, el mayor peso está en las industrias de procesos, que representaron, en promedio, el $50 \%$ del total en el lapso mencionado.

Con relación a las manufacturas de tecnología alta, se resalta el incremento de su participación dentro de las exportaciones totales (Tabla 2), lo cual es un aspecto positivo. Así, se pasó de un promedio del 6,5\% en 1995 a un 13,4\% en 2015. No obstante, como característica común de los países analizados, el peso de este tipo de manufacturas dentro de la estructura total continúa siendo bajo, incluso en países como la India, Turquía o Brasil.

La economía colombiana no fue ajena al fenómeno descrito hasta el momento. Este país pasó de exportar el 52,7\% en bienes de tecnología baja en 1995 a un 29,6\% en el 2015 (Tabla 2). Por el contrario, para las manufacturas de tecnología media los porcentajes fueron del $41,7 \%$ y $58,3 \%$, respectivamente. En el caso de las manufacturas de tecnología alta, se pasó de un porcentaje del 5,6\% en 1995 al 12,1\% en 2015. No obstante, a diferencia de la información encontrada para el resto de países, en los cuales la mayor participación está dada en las manufacturas relacionadas con productos eléctricos y electrónicos, en la economía colombiana se destaca el grupo de productos farmacéuticos, aeroespaciales, instrumentos ópticos de medición y cámaras. 
Teniendo en cuenta la clasificación de Lall (2000), quedan por evaluar aquellas exportaciones manufactureras que están basadas en recursos naturales, puesto que no quedan clasificadas en ninguna de las categorías tecnológicas que se mencionaron anteriormente. Para los 14 países analizados, la participación de este tipo de bienes representó, en promedio, el $53 \%$ de las exportaciones totales de manufacturas en el subperiodo 1995-2015. Incluso, dicho porcentaje tendió a incrementarse durante el periodo del boom de los precios de las commodities. Para algunos países como Argelia, Botswana, Chile y Nigeria el porcentaje es mucho más alto y se ubica por encima del $80 \%$ (Tabla 2). En contraste, Costa Rica y Turquía muestran una ratio más baja $(16 \%$, en promedio) y establecen una diferencia importante con relación al resto de países (Tabla 2 ).

En el caso de los países sudamericanos, las exportaciones de manufacturas basadas en recursos naturales representaron, en promedio, el $50 \%$ del total en el subperiodo 1995-2015 (Tabla 2). De estos países, el menor porcentaje está en Colombia (38\%) y el mayor en Chile (81\%).

En el caso de Colombia, se resalta que en el subperiodo 2010-2015, en el cual la influencia de los términos de intercambio en las exportaciones fue muy notoria, las manufacturas basadas en recursos naturales alcanzaron una participación promedio del $45 \%$ sobre el total de manufacturas (Tabla 2). Además, la mayor parte de ellas correspondieron a productos basados en otros recursos, como los minerales, el petróleo, el caucho, el cemento, etc., que aportaron, en promedio, el $70 \%$ del total de este grupo.

Con base en la información aportada, se concluye que no solamente se redujo la participación de las manufacturas dentro de los créditos de la cuenta corriente, fundamentalmente en aquellos países que mostraron una participación significativa de las exportaciones de recursos naturales, sino también que las características de las mismas no se han modificado radicalmente en términos de su componente tecnológico; y aunque se destaca la reducción de los productos de tecnología baja y el incremento de las manufacturas de tecnología media y alta, estas últimas todavía muestran una participación exigua en el total. 
Portillo-Riascos y Ortiz-Benavides: La vulnerabilidad externa de la economía colombiana...

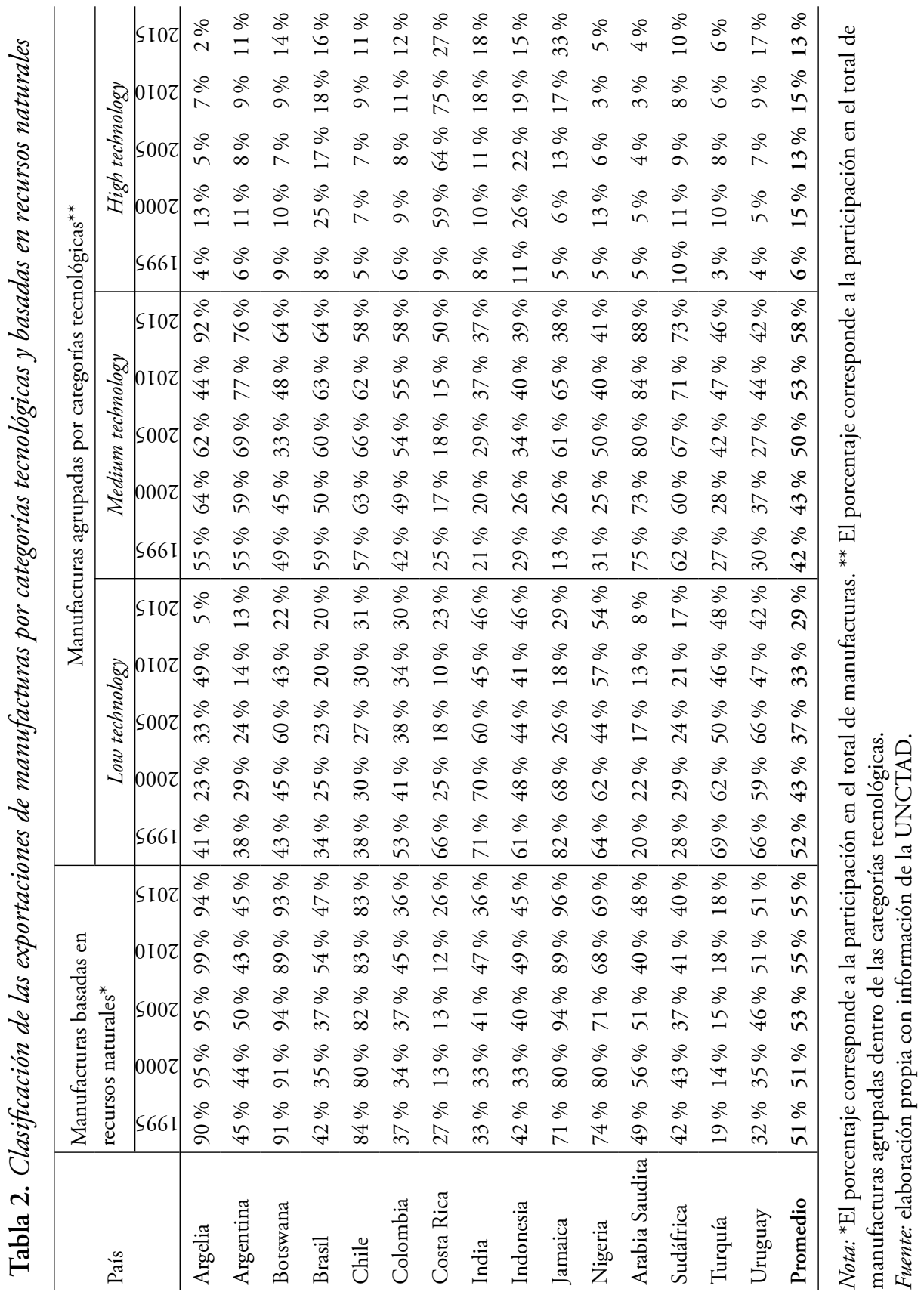


En el caso de los países sudamericanos estudiados, las exportaciones de manufacturas de tecnología alta alcanzaron un promedio del 13,4\% en 2015 (Tabla 2). Ahora bien, aunque este porcentaje es significativo teniendo en cuenta que en 1995 dicha ratio era del 5,78\%, lo más relevante es que gran parte de las exportaciones de manufacturas siguen basadas en recursos naturales, sin que se vislumbren mayores cambios dentro del periodo analizado. De hecho, como se observa en la Tabla 2, este tipo exportaciones pasó de representar el 51,8\% del total en 1995 al 55,1\% en 2015. En la economía colombiana sucedió algo similar: a pesar de que las exportaciones de manufacturas de alta tecnología pasaron del 6\% en 1995 al $12 \%$ en el 2015, la participación de las manufacturas en los créditos totales de la cuenta corriente se redujo del 17,5\% en 1990 al 16,2\% en el 2015.

Otra variable que está incorporada dentro del indicador de VR son las remesas enviadas por los emigrantes. Su importancia en cada uno de los países, desde luego, afecta el resultado de la medida de VR. Para el caso colombiano, si solamente se toman las exportaciones de manufacturas, la participación de estas dentro de los créditos totales de la cuenta corriente se reduce a un promedio del $22 \%$. Dicho resultado se explica porque las remesas reportaron una participación promedio del 11,2\% en el periodo 1990-2015. Ahora bien, al hacer la comparación con los otros cuatro países sudamericanos en este ítem, Colombia supera el promedio, que fue del 2,4\%. En estos términos, la VR de Colombia no solamente se explica porque este país mantiene un peso muy elevado de los ingresos derivados de las exportaciones de bienes primarios, sino también por la alta participación de las transferencias que realizan los emigrantes.

Una vez realizado el análisis de cada uno de los componentes de la VR, se realizó el comparativo que se muestra en la Figura 3. Nuevamente, es muy clara la tendencia hacia el incremento de la participación de los ingresos generados por las exportaciones de bienes primarios dentro de los créditos de la cuenta corriente. Entre los países sudamericanos seleccionados, el único caso donde no se vislumbra esta situación es en Brasil. En contraste, Colombia, Chile y Uruguay mostraron una mayor concentración de sus exportaciones respecto a este tipo de bienes. A diferencia de los países mencionados, es interesante la situación de India, Botswana, Turquía, 
Portillo-Riascos y Ortiz-Benavides: La vulnerabilidad externa de la economía colombiana...

Sudáfrica y Costa Rica, en los cuales se observa una mayor participación de los productos manufactureros.

Figura 3. Componentes del indicador de vulnerabilidad real

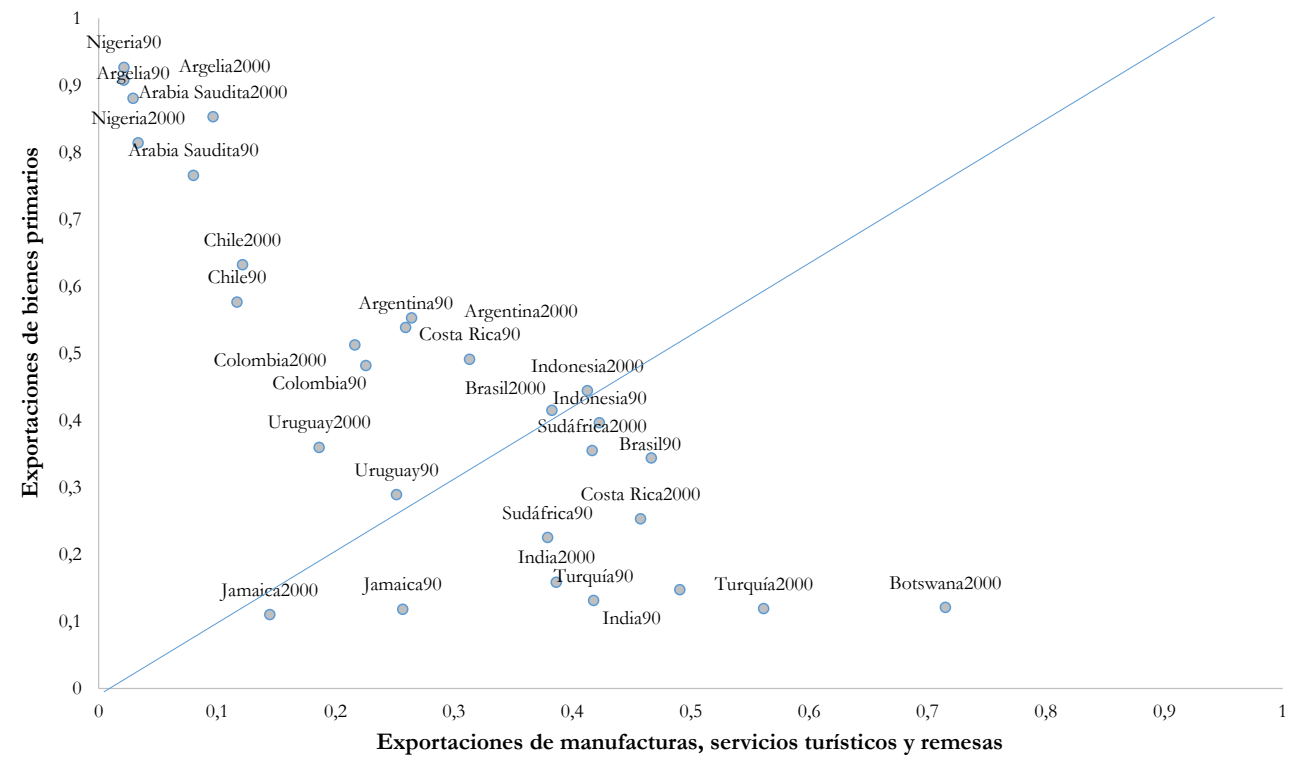

Fuente: elaboración propia con información de la CEPAL, el Banco Mundial, el FMI y las estadísticas oficiales de cada país.Los datos de la participación de la IED en el PIB se muestran en la Figura 4. Dado que la mayoría de los valores se ubican por encima de la diagonal, el resultado es que el peso de la IED se ha incrementado.

\section{B. Vulnerabilidad financiera (VF)}

Siguiendo la propuesta de Abeles y Valdecantos (2016), la VF de un país depende del stock de IED, el de IC (o la deuda externa pública), y el de reservas internacionales; expresados como porcentaje del PIB.

A partir de los resultados encontrados en este estudio, se concluye que la VF se incrementó en la mayoría de los países seleccionados. Este fenómeno se explica, fundamentalmente, por el crecimiento del stock de IED y, en menor medida, por el incremento de la IC. De igual manera, la deuda externa también 
muestra un saldo que, aunque se ha reducido, todavía conserva un peso significativo dentro de la estructura de pasivos.

Figura 4. Participación del stock de IED en el PIB

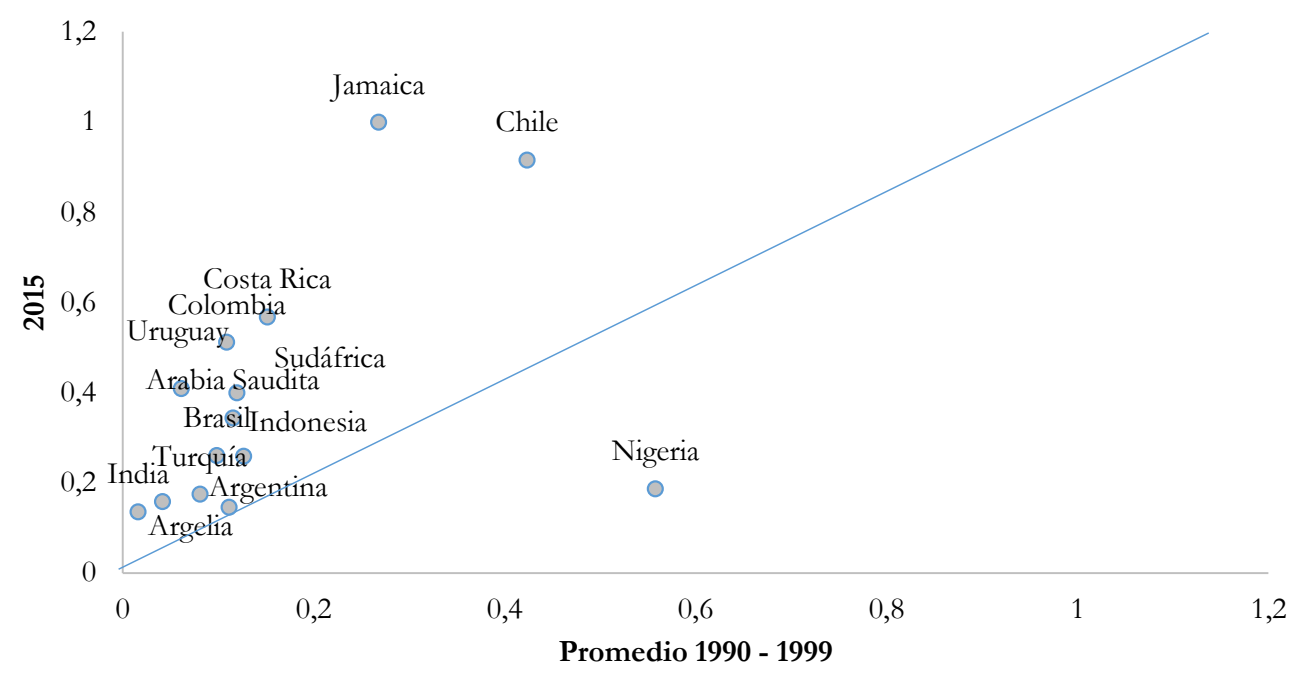

Fuente: elaboración propia con información de la CEPAL, el Banco Mundial, el FMI y las estadísticas oficiales de cada país.

Para el caso de Colombia, mientras en el subperiodo 1990-1999 el promedio de la participación del stock de IED dentro del PIB fue del $11 \%$, en el subperiodo 2000-2015 dicho porcentaje ascendió al 27,5\% promedio anual. Incluso, en 2015, dicha ratio alcanzó el 51,2\%. Si se hace el comparativo con los cuatro países sudamericanos seleccionados, el resultado de Colombia al cierre de 2015 solamente fue superado por Chile (91,5\%).

Con relación al stock de deuda externa pública (\% del PIB), en general, lo que se encontró es que hay una reducción para todos los países. En promedio, la ratio pasó del 33,9\% en el subperiodo 1990-1999 al 19,9\% entre 2000 y 2015, y se terminó el 2015 en un promedio del 15,2\%. Para el caso de Colombia, a pesar de haber reducido el peso de la deuda externa en el PIB, dicha ratio todavía se encuentra por encima de la media. En el contexto de los países sudamericanos seleccionados, al finalizar el 2015, a Colombia solamente la supera Uruguay. 
Portillo-Riascos y Ortiz-Benavides: La vulnerabilidad externa de la economía colombiana...

Respecto al stock de IC, en la mayoría de los países se incrementó la participación en el PIB. De hecho, se pasó de una media del 4,6\% en el subperiodo 1990-1999 a un porcentaje del 13,7\% entre 2000 y 2015, y se terminó en un promedio del 18,8\% al finalizar el 2015. En el caso de Colombia, la participación del stock de IC en el PIB siguió esta tendencia; no obstante, en el 2015 se ubicó por encima de la media y alcanzó un porcentaje del 21,4\%. Con respecto a los países sudamericanos seleccionados, los porcentajes son similares; excepto en el caso de Argentina (7,2\%).

Sobre el stock de reservas internacionales, que se entiende como una medida de protección ante episodios de salida masiva de capitales (autoseguro), en general, lo que se encontró es que, efectivamente, los países estudiados apuntaron hacia este objetivo. Por esta razón, se pasó de una media del $9 \%$ entre 1990 y 1999 a un porcentaje del 20,2\% en el subperiodo 2000 2015 , y se terminó con un promedio del $24,7 \%$ en el año 2015. En el caso de Colombia, se dio un fenómeno diferente, pues no hubo grandes diferencias en esta ratio entre los subperiodos 1990-1999 y 2000-2015; solamente entre el 2014 y el 2015 se presentó un cambio significativo, el cual se explica, más que por un incremento del stock de reservas, por una disminución del PIB nominal (expresado en dólares).

Los resultados de los cambios en la VF se muestran en la Figura 5. Llama la atención que, en promedio, el valor reportado para este indicador en el subperiodo 2000-2015 resultó inferior al del subperiodo 1990-1999, ya que pasó de un porcentaje del $43 \%$ al 34\%. Para explicar esta cifra, hay que tener en cuenta la situación de India, Botswana y Argelia, en los cuales la VF terminó siendo cercana a cero, o negativa. Eliminando estos tres países, la VF en el subperiodo 2000-2015, en promedio, fue del $48 \%$.

Para el caso de Colombia, que en el subperiodo 1990-1999 había mostrado un indicador de VF del 19\%, en el subperiodo 2000-2015 alcanzó un porcentaje del $34 \%$, e incluso, en el 2015, la VF fue del 57,9\%. Haciendo el comparativo con relación al resto de países sudamericanos, aunque la media de Colombia en el subperiodo 2000-2015 es inferior, al cierre de 2015 solamente es superada por Chile, cuyo indicador de VF alcanzó el 105,9\%. 
En estos términos, el comportamiento de la VF en el caso de Colombia resulta significativo respecto al resto de países seleccionados.

Figura 5. Cambios en la vulnerabilidad financiera por paises

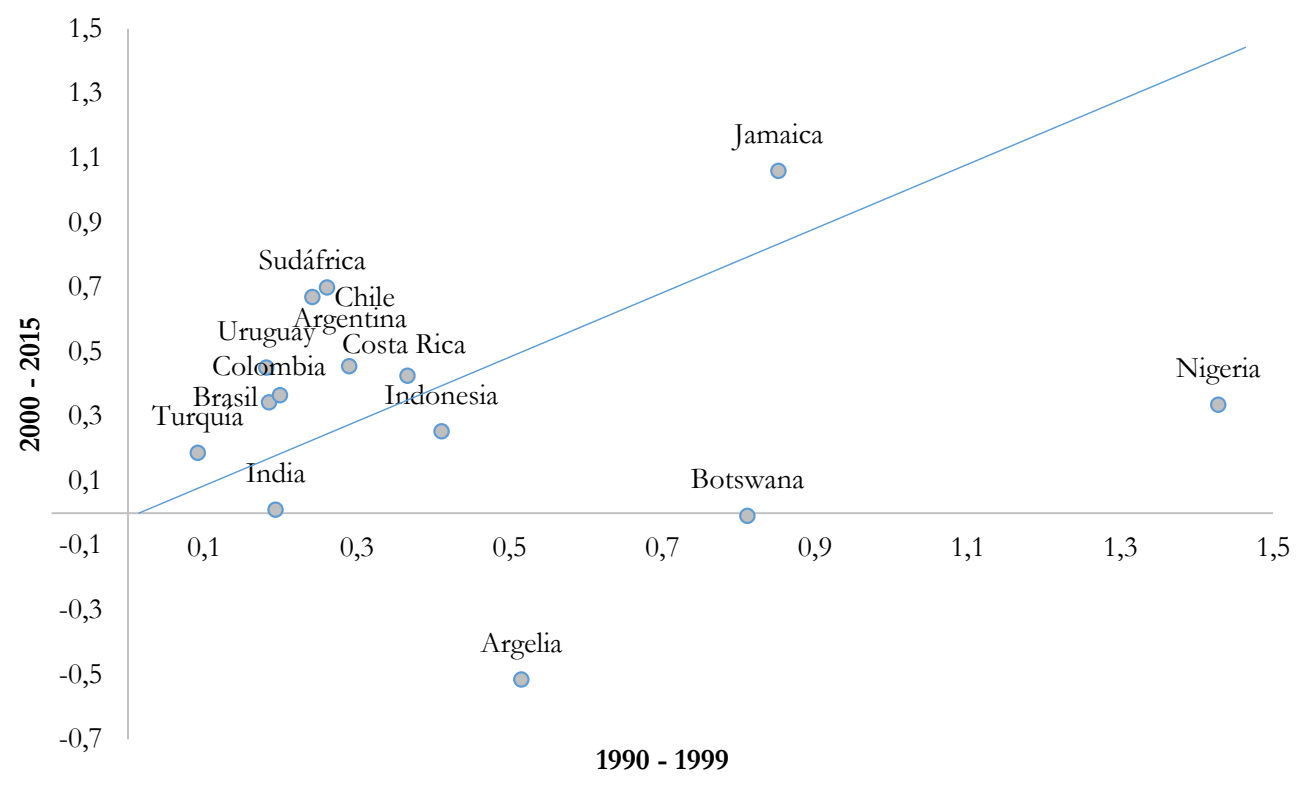

Fuente: elaboración propia con información de la CEPAL, el Banco Mundial, el FMI y las estadísticas oficiales de cada país.

\section{La vulnerabilidad externa}

A partir del análisis global del periodo estudiado, se concluye que el indicador que más creció para los países analizados fue la VF (Figura 6). Dicho fenómeno se explicó, fundamentalmente, por el comportamiento del stock de IED. Ahora bien, a pesar del incremento de la VF, la vulnerabilidad externa sigue explicándose por la VR (Figura 6). Así, en el subperiodo 2000-2015, la media del indicador de VR en la mayoría de países (10 de 14) es superior al resultado de la VF. La situación descrita también se puede observar en el caso de Colombia, ya que, al hacer el comparativo entre la década de 1990 y la del 2000, el resultado se ubica por debajo de la diagonal (Figura 6). 
Portillo-Riascos y Ortiz-Benavides: La vulnerabilidad externa de la economía colombiana...

El resultado general para Colombia es un incremento tanto de la VR como de la VF (Figura 6). Por tanto, a partir de estos dos indicadores, se puede afirmar que la vulnerabilidad externa de este país se incrementó a lo largo del periodo estudiado. Además, dichos resultados son significativos tanto en el contexto sudamericano como en el comparativo realizado con el resto de países seleccionados.

Figura 6. La vulnerabilidad externa por paises: comparativo del subperiodo 1990-1999 respecto al 2000-2015

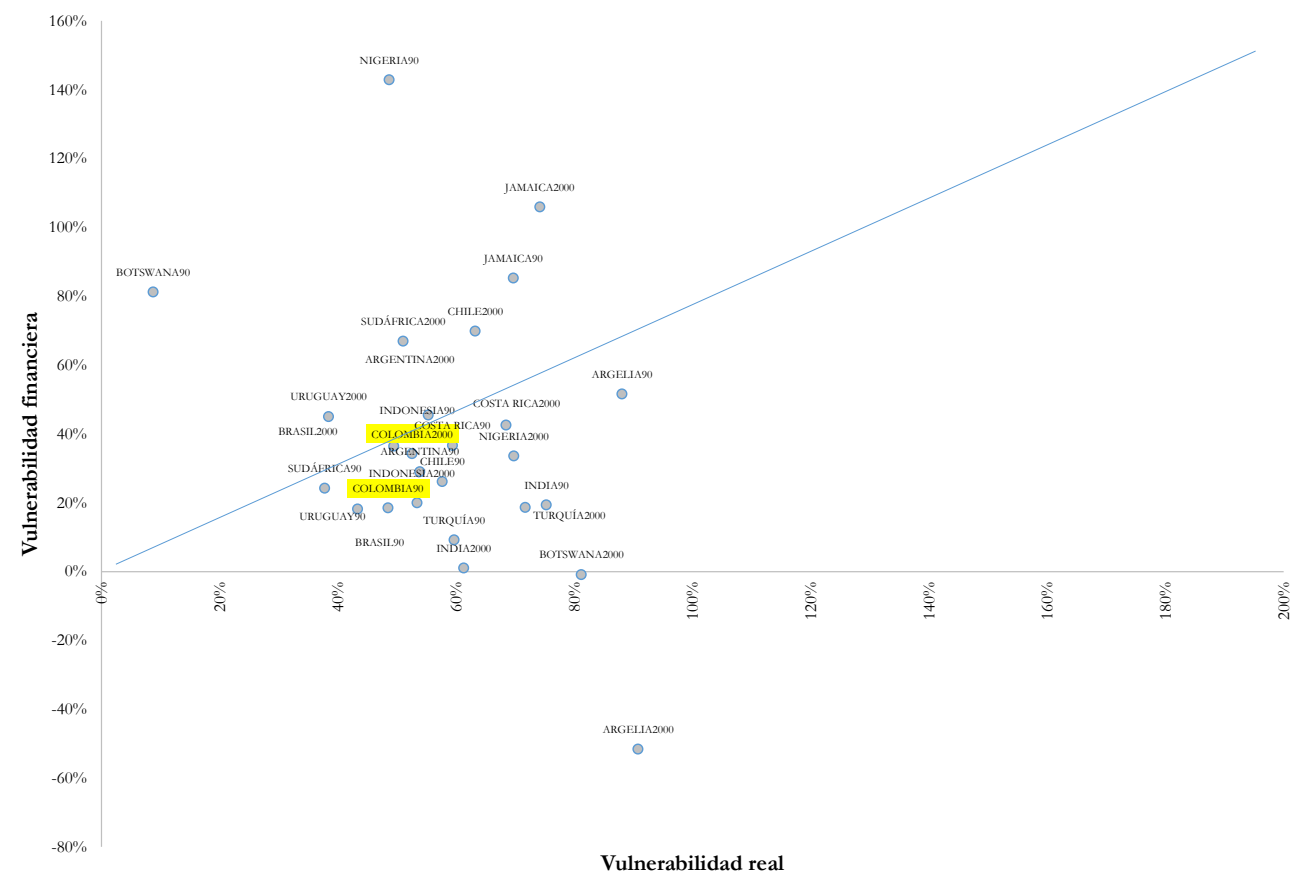

Fuente: elaboración propia con información de la CEPAL, el Banco Mundial, el FMI y las estadísticas oficiales de cada país.

A pesar de que no es un propósito de esta investigación, es interesante mencionar el comportamiento de los dos indicadores utilizados en este estudio durante el episodio de crisis económica de Colombia en el año 1999, así como también en la desaceleración que se presentó en este país el año 2009, como resultado de la crisis financiera de los países desarrollados. En 
los años previos a 1999, lo que se encontró es un crecimiento tanto de la VR como de la VF. En el primer caso, Colombia pasó de un porcentaje del $42 \%$ en 1993 al $51 \%$ en 1998, lo que representa un crecimiento del $20 \%$. Con relación a la VF, el incremento es del 100\%, desde el 13\% en 1994 al $26 \%$ en 2009. De esta manera, aunque el propósito de las dos medidas no es predecir la crisis, sí están mostrando una información relevante acerca de la vulnerabilidad externa de este país.

En cuanto al comportamiento de la VR y la VF en los años previos a 2009, lo que se encontró es que los dos indicadores se mantuvieron estables, y solamente en el momento en que se desencadenó la crisis en los países desarrollados mostraron cierta tendencia al crecimiento, especialmente en el caso de la VF, que pasó del $30 \%$ en 2008 al 38\% en 2009.

Los resultados encontrados dentro de este estudio, en general, son consecuentes con los hallazgos citados por Abeles y Valdecantos (2016). Sin embargo, hay tres diferencias importantes. La primera es que el periodo de referencia del presente estudio es más amplio, puesto que se toman un total de 26 años, mientras en el caso de Abeles y Valdecantos solamente se analizan 11 años. La segunda diferencia tiene que ver con el número y el tipo de países; así, mientras Abeles y Valdecantos examinan 32 países latinoamericanos, dentro de este estudio se analizan 14 países, incluyendo varios africanos y otros asiáticos, algunos con un modelo de inserción comercial similar al de Colombia y otros con una especialización productiva totalmente diferente. El tercer aspecto para resaltar es de tipo metodológico. De tal forma que, mientras Abeles y Valdecantos cuantifican la vulnerabilidad externa antes y después de la crisis financiera que se dio en varios países desarrollados en el lapso 2008-2009, dentro de este estudio las comparaciones se hacen entre la década de 1990 y la del 2000.

Más allá de las diferencias mencionadas, las conclusiones apuntan hacia un fortalecimiento de la vulnerabilidad externa, teniendo en cuenta la profundización del modelo de inserción comercial basado en la explotación de recursos naturales. Además, al incrementarse la dependencia con relación a la financiación externa, ya sea por la vía de la IED o de la IC, la sensibilidad de estas economías ante los cambios que se puedan dar en el contexto externo 
Portillo-Riascos y Ortiz-Benavides: La vulnerabilidad externa de la economía colombiana...

tiende a ser mayor; tal y como viene ocurriendo desde el 2013, cuando los precios de las commodities empezaron a reducirse.

\section{Conclusiones}

El objetivo de este estudio fue cuantificar la vulnerabilidad externa de la economía colombiana para el periodo 1990-2015. Además, se buscó hacer un comparativo de los resultados mostrados para este indicador en este país con un conjunto de economías, algunas con un modelo de especialización productiva similar y otras con una estructura de inserción comercial completamente diferente.

A partir de los resultados encontrados, se concluye que la economía colombiana incrementó tanto la VR como la VF. Además, se colige que este es un resultado relevante en el contexto sudamericano, así como en el comparativo realizado con el resto de los países seleccionados. En consecuencia, se puede afirmar que la vulnerabilidad externa de la economía colombiana se intensificó a lo largo del periodo estudiado. Esta contribución es importante porque muestra un indicador concreto de vulnerabilidad externa, que, aunque tiene ciertas falencias y limitaciones, muestra varios de los elementos estructurales que hacen parte de dicho fenómeno.

El incremento de la vulnerabilidad externa de la economía colombiana se explica por dos razones. En primer lugar, por el aumento de la participación de las exportaciones de bienes primarios dentro de la estructura de los créditos de la cuenta corriente, y la consecuente reducción del peso de las manufacturas. Además, se fortaleció la importancia de las remesas, cuya dinámica desde finales de los noventa ha llevado a que se conviertan en la segunda fuente de financiamiento externo más importante de la economía colombiana después de la IED.

En segundo lugar, la economía colombiana incrementó su dependencia respecto al financiamiento externo, evidenciada en el mayor peso del stock de IED y de la IC. Dicho fenómeno se ha visto reflejado en una mayor presión de las rentas factoriales dentro de la estructura de la cuenta corriente, especialmente en aquellos años en los cuales se dio un crecimiento 
significativo de las rentas de los recursos naturales (2003-2013). Ahora bien, aunque en el periodo analizado se dio un incremento importante del stock de reservas internacionales, especialmente durante los primeros años de la década de los 2000, su participación en el PIB se ha mantenido en niveles similares a los reportados en la década de los noventa.

En suma, lo que se pudo mostrar a partir de este estudio es que la economía colombiana no ha sido capaz de hacer un cambio positivo en su patrón de inserción comercial. Por el contrario, lo que se ha dado es un fortalecimiento del modelo de especialización productiva basado en la explotación de los recursos naturales, fenómeno que explica el incremento de la vulnerabilidad externa de este país. Ahora bien, lo que se pudo ver es que este fenómeno no solamente se ha dado en Colombia, sino también en el resto de los países sudamericanos seleccionados.

Así entonces, desde la perspectiva neoestructuralista, las razones estructurales que explican la vulnerabilidad externa de este tipo de países no han cambiado, e incluso han tendido a reforzarse desde la fase de profundización de la apertura comercial y financiera que se dio desde principios de los noventa.

\section{Agradecimientos}

Este artículo toma algunos apartes de la investigación intitulada "Vulnerabilidad e inestabilidad macroeconómica de Colombia en el periodo 1990-2015", cuya memoria fue presentada por Luis Hernando Portillo Riascos para optar al título de Doctor en Economía por la Universidad Complutense de Madrid (año 2019).

\section{Referencias}

Abeles, M. \& Valdecantos, S. (2016). Vulnerabilidad externa en América Latina y el Caribe. Un análisis estructural (Serie Estudios y perspectivas, No. 49). Comisión Económica para América Latina y el Caribe. https: //www.cepal.org/es/publicaciones/40502-vulnerabilidad-externa-amer ica-latina-caribe-un-analisis-estructural 
Portillo-Riascos y Ortiz-Benavides: La vulnerabilidad externa de la economía colombiana...

Angeon, V. \& Bates, S. (2015). Reviewing composite vulnerability and resilience indexes: a sustainable approach and application. World Development, 72, 140-162.

Atkins, J., Mazzi, S. \& Ramlogan, C. (1998). A study of the vulnerability of developing and island states: a composite index. Commonwealth Secretariat.

Bates, S., Angeon, V. \& Ainouche, A. (2014). The pentagon of vulnerability and resilience: A methodological proposal in development economics by using graph theory. Economic Modelling, 42, 445-453.

Bielschowsky, R., Izam, M. \& Mulder, N. (2011). Dos estudios de la evolución del pensamiento de la CEPAL sobre la diversificación productiva y la inserción internacional (1949-2008) (Series Comercio Internacional, No. 112). Comisión Económica para América Latina y el Caribe. https: //www.cepal.org/es/publicaciones/4337-estudios-la-evolucion-pe nsamiento-la-cepal-la-diversificacion-productiva-la

Briguglio, L. (1995). Small island developing states and their economic vulnerabilities. World Development, 23(9), 1615-1632.

Briguglio, L. (2003). The vulnerability index and Small Island Developing States: A review of conceptual and methodological issues. https://pdfs.semanticsch olar.org/add1/0617fdab11e938a236a1ec10cb67b32abae9.pdf

Briguglio, L. (2014). A vulnerability and resilience framework for small states. In Bynoe-Lewis, D. (Ed.), Building the resilience of small states: a revised framework (pp. 1-102). Commonwealth Secretariat.

Briguglio, L., Cordina, G., Farrugia, N. \& Vella, S. (2009). Economic vulnerability and resilience: concepts and measurements. Oxford Development Studies, 37(3), 229-247.

Busso, G. (2001). Vulnerabilidad social: nociones e implicancias de politicas para Latinoamérica a inicios del siglo XXI. http://www.derechoshumanos.un lp.edu.ar/assets/files/documentos/vulnerabilidad-social-nociones-e-imp licancias-de-politicas-para-latinoamerica-a-inicios-del-siglo-xxi.pdf 
CEPAL (Comisión Económica para América Latina y el Caribe). (2012). Cambio estructural para la igualdad: una visión integrada del desarrollo. Trigésimo cuarto periodo de sesiones de la CEPAL. https://repositorio.cepa 1.org/bitstream/handle/11362/3078/1/S2012062_es.pdf

CEPAL (Comisión Económica para América Latina y el Caribe). (2016). Horizontes 2030: la igualdad en el centro del desarrollo sostenible. https: //www.cepal.org/es/publicaciones/40159-horizontes-2030-la-igualdad -centro-desarrollo-sostenible

Chambers, R. (1989). Vulnerability: how the poor cope. IDS Bulletin, 20(2), $1-7$.

Cordina, G. (2004). Economic vulnerability and economic growth: some results from a neo-classical growth modelling approach. Journal of Economic Development, 29(2), 21-39.

Da Costa, V. \& Libânio, G. (2013). Volatilidad de los flujos financieros y fuga de capitales: la vulnerabilidad externa de Brasil, 1995-2010. Investigación Económica, 72(286), 65-100.

Dabla-Norris, E. \& Gündüz, Y. (2014). Exogenous choques and growth crises in low-income countries: a vulnerability index. World Development, 59, 360-378.

Domínguez, M., Blancas, F., Guerrero, F. \& González, M. (2011). Una revisión crítica para la construcción de indicadores sintéticos. Métodos Cuantitativos para la Economía y la Empresa, 11, 41-70.

Essers, D. (2013). Developing country vulnerability in light of the global financial crisis: Shock therapy? Review of Development Finance, 3(2), 61-83.

Ffrench-Davis, R. (2008). From financieristic to real macroeconomics: Seeking development convergence in emerging economies (Commission on growth and development working paper, No. 44). World Bank. https://docume nts.worldbank.org/en/publication/documents-reports/documentdetail/ 642921468348846547/from-financieristic-to-real-macroeconomics-se eking-development-convergence-in-emerging-economies 
Portillo-Riascos y Ortiz-Benavides: La vulnerabilidad externa de la economía colombiana...

Ffrench-Davis, R. (2009). Políticas macroeconómicas para el desarrollo en América Latina. CIDOB d'Afers Internacionals, 85-86, 109-131.

Ffrench-Davis, R. (2010). Macroeconomía para el desarrollo: desde el financierismo al productivismo. Revista de la CEPAL, 102, 7-27.

Ffrench-Davis, R. (2015). Neoestructuralismo y macroeconomía para el desarrollo. En A. Bárcena \& A. Prado (Eds.), Neoestructuralismo y Corrientes heterodoxas en América Latina y el Caribe a inicios del siglo XXI (pp. 119-143). CEPAL.

FMI (Fondo Monetario Internacional) (1998). Financial crises: characteristics and indicators of vulnerability. https://www.imf.org/external/pubs/ft/w eo/weo0598/

FMI (Fondo Monetario Internacional) (2010). The IMF-FSB Early warning exercise. Design and methodological toolkit. https://www.imf.org/external /np/pp/eng/2010/090110.pdf

FMI (Fondo Monetario Internacional) (2011). Managing volatility: a vulnerability exercise for low-income countries. https://www.imf.org/ex ternal/np/pp/eng/2011/103111a.pdf

Frankel, J. \& Saravelos, G. (2012). Can leading indicators assess country vulnerability? Evidence from the 2008-09 global financial crisis. Journal of International Economics, 87(2), 216-231.

Ghosh, S. \& Ghosh, A. (2003). Structural vulnerabilities and currency crises. IMF Economic Review, 50(3), 481-506.

Gonçalves, R., Carcanholo, M., Filgueiras, L. \& Pinto, E. (2009). Vulnerabilidad estructural externa en América Latina. En E. Arceo \& E. Basualdo (Eds.), Los condicionantes de la crisis en América Latina: inserción internacional y modalidades de acumulación (pp. 119-138). CLACSO.

Guillaumont, P. (2009). An economic vulnerability index: its design and use for international development policy. Oxford Development Studies, 37(3), 193-228. 
Guillaumont, P. (2010). Assessing the economic vulnerability of small island developing states and the least developed countries. The Journal of Development Studies, 46(5), 828-854.

Guillaumont, P. (2013). Measuring structural vulnerability to allocate development assistance and adaptation resources (Development Policies Working Paper, No. 68). Fondation pour les Études et Recherches sur le Développement International. https://ferdi.fr/en/publications/measu ring-structural-vulnerability-to-allocate-development-assistance-and-a daptation-resources

Guillaumont, P. (2016). Vulnerability and resilience: a conceptual framework applied to three Asian countries Bhutan, Maldives and Nepal (ADB South Asia Working Paper Series, No. 53). Asian Development Bank, Philippines. https://www.adb.org/publications/vulnerability-resiliencebhutan-maldives-nepal

Herrera, S. \& Garcia, C. (1999). User's guide to an early warning system for macroeconomic vulnerability in Latin American countries. Journal of Economics, 98(4), 463-484.

Kaminsky, G., Lizondo, S. \& Reinhart, C. (1998). Leading indicators of currency crises (Staff Papers, Vol. 45, No. 1). International Monetary Fund. https://www.imf.org/en/Publications/WP/Issues/2016/12/ 30/Leading-Indicators-of-Currency-Crises-2256

Lall, S. (2000). The Technological structure and performance of developing country manufactured exports, 1985-98. Oxford Development Studies, 28(3), 337-369.

Le Fort, G. \& Budnevich, C. (2005). Entendiendo las volatilidades cíclicas de la cuenta de capitales y del PIB: un estudio de panel para países latinoamericanos (Serie Macroeconomía del Desarrollo, No. 42). CEPAL. https://www.cepal.org/es/publicaciones/5408-entendiendo-volatilidade s-ciclicas-la-cuenta-capitales-pib-un-estudio-panel

Loayza, N. \& Raddatz, C. (2007). The structural determinants of external vulnerability. The World Bank Economic Review, 21(3), 359-387. 
Portillo-Riascos y Ortiz-Benavides: La vulnerabilidad externa de la economía colombiana...

Montalbano, P. (2011). Trade openness and developing countries vulnerability: concepts, misconceptions, and directions for research. World Development, 39(9), 1489-1502.

Ocampo, J. A. (2001). Raúl Prebisch y la agenda del desarrollo en los albores del siglo XXI. Revista de la CEPAL, 75, 25-40.

Ocampo, J. A. (2008). El auge económico latinoamericano. Revista de Ciencia Politica, 28(1), 7-33.

Ocampo, J. A. (2011). Macroeconomía para el desarrollo: políticas anticíclicas y transformación productiva. Revista de la CEPAL, 104, 13-41.

Ocampo, J. A. (2012). Balance of payments dominance: Its implications for macroeconomic policy (Unpublished paper). School of International and Public Affairs, Columbia University.

Portillo-Riascos, L. H. (2020a). Los orígenes de la vulnerabilidad macroeconómica y sus principales efectos: propuesta de un marco teórico. Iberoamerican Journal of Development Studies, 9(2), 208-229. https://doi.org/10.26754/ojs_ried/ijds.474

Portillo-Riascos, L. H. (2020b). El concepto de vulnerabilidad macroeconómica. Una revisión de la literatura. Sociedad y Economía, 40, 99-118. https://doi.org/10.25100/sye.v0i40.8020

Prebisch, R. (1949). El desarrollo económico de la América Latina y algunos de sus principales problemas. CEPAL. https://www.cepal.org/es/publicacion es/40010-desarrollo-economico-la-america-latina-algunos-sus-principa les-problemas

Rodríguez, O. (2006). El estructuralismo latinoamericano. Siglo XXI Editores.

Seth, A. \& Ragab, A. (2012). Macroeconomic vulnerability in developing countries: Approaches and issues (Working Paper, No. 94). International Policy Centre for Inclusive Growth. https://ipcig.org/pub/IPCWorking Paper94.pdf 
Schuschny, A. \& Soto, H. (2009). Guía metodológica: diseño de indicadores compuestos de desarrollo sostenible. CEPAL. https://www.cepal.org/es/p ublicaciones/3661-guia-metodologica-diseno-indicadores-compuestosdesarrollo-sostenible

Titelman, D., Pérez, E. \& Minzer, R. (2008). Comparación de la dinámica e impactos de los choques financieros y de términos del intercambio en América Latina en el periodo 1980-2006 (Serie Financiamiento del Desarrollo, No. 203). CEPAL. https://www.cepal.org/es/publicaciones/5180-comparac ion-la-dinamica-impactos-choques-financieros-terminos-intercambioamerica

Thirlwall, A. (1979). The balance of payments constraint as an explanation of international growth rates differences. BNL Quarterly Review, 32(128), 45-53.

Thirlwall, A. (2011). Balance of payments constrained growth models: history and overview. PSL Quarterly Review, 64(259), 307-351.

Valdecantos, S. (2016). Estructura productiva y vulnerabilidad externa: un modelo estructuralista stock-flujo consistente (Serie Estudios y Perspectivas, No. 46). CEPAL. https://www.cepal.org/es/publicaciones/40094-estru ctura-productiva-vulnerabilidad-externa-un-modelo-estructuralista-st ock

Villagrán, J. C. (2006). Vulnerability: a conceptual and methodological review. http://collections.unu.edu/eserv/unu:1871/pdf3904.pdf 